\title{
Effect of SPAN80 on the structure of emulsified aqueous suspensions
}

\author{
M. Schmitt ${ }^{1}$, S. Limage ${ }^{2}$, R. Denoyel ${ }^{1}$ and M. Antoni ${ }^{1}$ \\ ${ }^{1}$ Aix-Marseille Univ, CNRS, Marseille, France \\ ${ }^{2}$ Genes'Ink, Rousset, France
}

\begin{abstract}
The influence of an oil-soluble surfactant (SPAN80) on water in oil emulsions is investigated. The aqueous phase is a nanofluid consisting in a suspension of silica nanoparticles modified by CTAB. This aqueous solution, when emulsified in paraffin oil, is known to produce droplets with structural properties that depend upon the $[\mathrm{CTAB}] /\left[\mathrm{SiO}_{2}\right]$ mixing ratio. For large mixing ratios, droplets have the usual spherical shape whereas for small ones, they are deformed and behave like stiff polymorphous objects. The present work focuses on the study of the robustness of this phenomenon when adding SPAN80 into the paraffin oil phase in a broad range of concentrations. Optical tomography microcopy is used to describe the structure of the emulsions. SPAN80 actually comes in addition to the stabilizing role of CTAB. It contributes in the decrease of interfacial tension and to a larger dispersity. For sufficiently large concentrations, emulsions are shown to always contain spherically shaped droplets. A similar phenomenology is observed for SDS/alumina aqueous suspensions. This suggests that the change from polymorphous to spherically shaped droplets is a general property of emulsified nanofluids in SPAN80/paraffin oil solutions.
\end{abstract}

Keywords: Emulsions, Nanofluids, Nanoparticles, Surfactants, Structure, Optical tomography.

\section{Introduction}

Emulsions are widely used in many fundamental and applied research fields such as biomedical materials, pharmaceuticals, cosmetics, coatings and food ${ }^{1-5}$. They can be classified into different groups depending upon their stabilization mechanisms that result from either surface active molecules, solid particles or a combination of both ${ }^{5-}$ 10. For particle-less emulsions like for example nanoemulsions, remarkable pharmacological applications can be produced with low energy emulsification procedures ${ }^{11}$. It is also known for more than a century that emulsions can be stabilized by particles and that such systems, called Ramsden-Pickering emulsions, involve complex adsorption and wetting mechanisms ${ }^{12-14}$. Despite a longstanding knowledge, the understanding of Ramsden-Pickering emulsions is still subject to an intense research activity. Their stabilization is connected to the adsorption kinetics of particles into interfacial layers driven by particle hydrophobicity. Important studies have been devoted to this problem in the past years with a special emphasis on the interplay between emulsion stability and interfacial properties ${ }^{15-19}$. An interesting property of surfactantnanoparticles aqueous suspensions, denoted as surfactant modified nanofluids (SMNs) hereafter, is the possibility to tune wetting properties and to control both nanoparticles adsorption at interfaces and nanoparticle aggregation. Such synergistic effects are known to stabilize emulsions and are controlled by $\mathrm{pH}$ and electrolyte strength ${ }^{20-22}$.

This article presents experimental results involving nanoparticles interactions in SMNs emulsified in an organic phase with varying nanoparticle and surfactant concentrations. The volume fraction of the SMNs is small (1 $\%)$. Emulsions are therefore dilute and remain optically transparent when using a soft emulsification protocol like magnetic stirring. The continuous organic phase is a mixture of paraffin oil (PO) and SPAN80 while the dispersed phase is a SMN solution consisting in most of this work in water, silica nanoparticles and CTAB. Sodium dodecyl sulfate/alumina nanoparticles SMNs will also be discussed. All these emulsions are known to exhibit different structures depending upon the surfactant/nanoparticles mixing ratio. For $\mathrm{CTAB} / \mathrm{SiO}_{2}$ mixtures, it is defined by $\mathrm{R}=[\mathrm{CTAB}] /\left[\mathrm{SiO}_{2}\right]$ ${ }^{23-26}$. When $\mathrm{R}>0.03$, the droplets take a usual spherical shape as illustrated in Figure 1(a) while for $\mathrm{R}<0.03$ they show polymorphous shapes similar to the ones represented in Figure 1(b). The driving mechanism of this phenomenon is a modification of the coverage of the nanoparticles by CTAB that allows the appearance of $\mathrm{SiO}_{2}$ microstructures ${ }^{23}$. $\mathrm{R}=0.03$ is indeed close to the isoelectrical point where the zeta potential vanishes and nanoparticles start to flocculate. When deformed, droplets behave as stiff objects and do neither exhibit shape changes nor coalescence even when the emulsions drain and droplet come into contact ${ }^{26}$. The properties of such emulsions have been investigated with optical tomography microscopy and scanning electron microscopy $^{25,28}$. Bulk rheology measurements on water/CTAB $/ \mathrm{SiO}_{2}$ mixtures have also been achieved and demonstrated either newtonian or viscoelastic behaviors. Viscoelasticity was shown to result from the formation of $\mathrm{SiO}_{2}$ microstructures ${ }^{28}$. Similar emulsions were studied but with a continuous phase consisting of hexane or hexadecane ${ }^{24,29}$.

Polymorphous droplet emulsions are very stable against coalescence. This is interpreted as a consequence of the formation of solid-like interfacial layers ${ }^{29}$. This hypothesis was confirmed by interfacial rheology characterizations of $\mathrm{CTAB} / \mathrm{SiO}_{2}$ mixtures where an irreversible attachment of the nanoparticles at the interfaces and the formation of solid-like films have been evidenced 27,30-33. Similar properties have been observed when replacing CTAB and $\mathrm{SiO}_{2}$ nanoparticles by sodium dodecyl sulfate (SDS) and alumina nanoparticles ${ }^{34}$. This suggests that the occurrence of emulsions with spherical/polymorphous droplets is probably a general 
feature in emulsified suspensions. One still open question in this context is the stability of the above solid-like films at liquid/liquid interfaces and emulsion structure when adding oil soluble surfactants. Interfacial rheology of SPAN80 adsorbed at water/paraffin oil interfaces and its effect on water in paraffin-oil emulsions demonstrate the major role played by dilational elasticity. This gives an interesting direction for the understanding of droplet deformation ${ }^{27}$.

The goal of this work is to investigate the specific influence SPAN80 in emulsified dispersions for a broad range of concentrations. Optical tomography microscopy analysis and imaging will be used. Many different surfactant/nanoparticles mixtures could have been studied here. Some results on SDS/Alumina SMNs will be rapidly presented but the detailed analysis will focus on $\mathrm{CTAB} / \mathrm{SiO}_{2}$ ones. This article is divided into five sections. After this introduction, section 2 is devoted to materials and methods. The preparation of the SMNs and the emulsification protocol are described. Section 3 focuses on the image processing technique and introduces a criterion to identify the structure of the emulsions. Section 4 is devoted to the results and discussions. Conclusions and perspectives are finally drawn in section 5 .

\section{Materials and methods}

Emulsions are formulated in two steps. SMNs with suitable $\mathrm{SiO}_{2}$ concentrations are first prepared diluting a commercial aqueous colloidal silica suspension (Levasil@ 200/30\%, supplied by Stark GmbH/ Germany) with aqueous solutions of hexadecyltrimethylammonium bromide (CTAB, Fluka ultra grade $52365, \mathrm{CMC} \approx 0.36 \mathrm{~g} / \mathrm{L}$ ) and water (HPLC grade). Levasil@ is highly alkaline $(\mathrm{pH} \approx 9.2)$ and the radius of the nanoparticles is about 7,5 nm for a surface area of 200 $\mathrm{m}^{2} / \mathrm{g}$. Due to high hydrophobicity, this dispersion shows an excellent stability over months. This is the result of the very negative surface potential of the nanoparticles that promote electrostatic repulsion $(-40 \mathrm{mV})$. When diluting it with CTAB aqueous solutions, a decrease of $\mathrm{pH}$ is observed. It starts from the above value and relaxes to a stable final one after 4 to 5 hours. This time is required for neutralization of the surface charge of the nanoparticles by the CTAB cations. In the conditions of this work, the final $\mathrm{pH}$ remains always above the gellation point $(\mathrm{pH}=5.5)$. Both $\mathrm{SiO}_{2}$ and $\mathrm{CTAB}$ concentrations are varied in a way to produce SMNs with controlled interfacial properties. These latter are ultimately emulsified in paraffin oil solutions (Fluka 76235) which have been used without further purification and prepared with varying SPAN80 (Fluka 09569, CMC $=0.4310^{-3} \mathrm{~mol} / \mathrm{L}$ in PO) concentrations ${ }^{27}$. The concentrations of the chemicals used for the $\mathrm{CTAB} / \mathrm{SiO}_{2} / \mathrm{SPAN} 80$ emulsions that will be studied hereafter are given in table 1 .

\begin{tabular}{|c|c|c|c|c|}
\hline Name & $\begin{array}{c}{[\mathrm{CTAB}]} \\
(\mathrm{g} / \mathrm{L})\end{array}$ & $\begin{array}{c}{\left[\mathrm{SiO}_{2}\right]} \\
(\mathrm{g} / \mathrm{L})\end{array}$ & $\begin{array}{c}{[\mathrm{SPAN} 80]} \\
(\mathrm{g} / \mathrm{L})\end{array}$ & $\mathrm{R}$ \\
\hline $\mathrm{S} 1$ & 0.219 & 12 & 0 & $1.810^{-2}$ \\
\hline $\mathrm{S} 2$ & 0.219 & 12 & $4.2510^{-2}$ & $1.810^{-2}$ \\
\hline $\mathrm{S} 3$ & 0.219 & 120 & 0 & $2.10^{-3}$ \\
\hline $\mathrm{S} 4$ & 0.219 & 120 & $4.2510^{-2}$ & $2.10^{-3}$ \\
\hline $\mathrm{S} 5$ & 2.19 & 120 & 0 & $1.810^{-2}$ \\
\hline $\mathrm{S} 6$ & 2.19 & 120 & $4.2510^{-2}$ & $1.810^{-2}$ \\
\hline
\end{tabular}

Table 1 : Composition of the emulsions of Figure 4 together with the values the mixing ration $\mathrm{R}$.
The same protocol is followed for the production of SDS/alumina SMNs. Alumina nanoparticles (Disperal powder supplied by the producer Sasol Germany) are mixed with SDS, an anionic surfactant (anionic surfactant Sigma Aldrich, 99\%) and water (HPLC grade). According to the data provided by Sasol Germany ${ }^{35}$, Disperal is a synthetic boehmite alumina system produced from aluminum alkoxides. It is a dispersible powder composed of crystallites of $10 \mathrm{~nm}$. Alumina content is approximately $77 \%$, indicating slightly higher water concentrations than in the stoichiometric boehmite $[\mathrm{AlO}(\mathrm{OH})]$. In an acidic medium, this dispersion exhibits excellent long-term stability due to the dissociation of two hydroxyl groups generating a large positive surface charge with a zeta-potential close to +45 $\mathrm{mV}$. The concentrations of the chemicals used for the SDS/Disperal/SPAN80 emulsions that will be studied hereafter are given in table 2.

\begin{tabular}{|c|c|c|c|c|}
\hline Name & $\begin{array}{c}{[\mathrm{SDS}]} \\
(\mathrm{g} / \mathrm{L})\end{array}$ & $\begin{array}{c}{[\text { Disperal }]} \\
(\mathrm{g} / \mathrm{L})\end{array}$ & $\begin{array}{c}{[\text { SPAN80] }} \\
(\mathrm{g} / \mathrm{L})\end{array}$ & $\mathrm{R}$ \\
\hline $\mathrm{S}^{\prime}{ }^{\prime}$ & 0.01 & 5 & 0 & $2.10^{-3}$ \\
\hline $\mathrm{S}^{\prime}$ & 0.01 & 5 & 0.1 & $2.10^{-3}$ \\
\hline $\mathrm{S}^{\prime}{ }^{\prime}$ & 0.01 & 25 & 0 & $4.10^{-4}$ \\
\hline $\mathrm{S}^{\prime}{ }^{\prime}$ & 0.01 & 25 & 0.1 & $4.10^{-4}$ \\
\hline $\mathrm{S}^{\prime}$ & 0.1 & 25 & 0 & $4.10^{-3}$ \\
\hline $\mathrm{S}^{\prime}$ & 0.1 & 25 & 0.1 & $4.10^{-3}$ \\
\hline
\end{tabular}

Table 2: Composition of the emulsions of Figure 5 with the values the mixing ration $R$.

Experiments are performed at room temperature and the volume fraction of all emulsions is fixed to $1 \%$. Emulsification is achieved according to the following protocol: SMNs are first introduced in the PO/SPAN80 solution with a syringe in a $50 \mathrm{~mL}$ beaker. Both phases are initially separated. The total volume of solution at this stage of the procedure is $30 \mathrm{~mL}$. Due to the high viscosity of PO, the SMN droplet is floating right after introduction in the beaker. This system is degassed for $10 \mathrm{~min}$ under vacuum conditions. Emulsification is then achieved by magnetic stirring for $10 \mathrm{~min}$ at $800 \mathrm{rpm}$ with a cylindrical stirring bar (1 cm long, $0.5 \mathrm{~cm}$ diameter). The main interest of this protocol is to produce, for the CTAB, SDS and SPAN80 concentrations ranges of Tables 1 and 2, transparent emulsions and to ensure a good reproducibility of the experiments. Finally, $4 \mathrm{~mL}$ of the resulting emulsions are poured into an optical quartz cell (Hellma, 110-QS) and analyzed by optical tomography microscopy (OTM). Each emulsion was produced three times and three OTM shots were performed. All experiments show a good reproducibility in the emulsion properties although the obtained images are always different.

OTM combines a classical microscope in transmitted light mode with a CCD camera (Microtron MC1310) and an objective attached to a moving translation stage. This technique is nonintrusive and allows in situ observations of the structure of the emulsions at distances larger than $1 \mathrm{~mm}$ from the front wall of the quartz cells. Influence of boundary conditions on the overall structure of the emulsion can therefore be considered as negligible. A single tomographic shot takes $1 \mathrm{~s}$ and corresponds to the scanning of a $1 \mathrm{~mm}^{3}$ volume. Over such short periods of time, emulsions are almost at rest. Successive images remain therefore coherent and allow a reliable detection of each droplet. Several hundreds of droplets can be tracked. 500 images encoded with $1024 \times 1024$ pixels are produced, each 
of them showing a $1 \mathrm{~mm}^{2}$ field of view. Spatial resolution is therefore about $1 \mu \mathrm{m}$. In the scanning direction spatial resolution is $5 \mu \mathrm{m}$. Finally, the depth of field of the microscope is about $10 \mu \mathrm{m}$.

Isotherms are deduced from surface tensions that are measured with the Du Nouÿ method using a high sensitive balance. In this technique a ring is put in contact with the liquid/air interface and used to measure the change of force when pulling it apart from the interface. The measured first force maximum is used to estimate the surface tension. Each data point has been measured at least five times to ensure reproducibility. The interfacial tension is evaluated only for air-CTAB/SiO ${ }_{2}$ SMNs interfaces as a function of added CTAB. For a given interfacial tension, the difference of added CTAB between an experiment with silica and without silica gives the amount of adsorbed CTAB. The excess surface concentration $\Gamma$ is then evaluated from the mass of silica in the SMN and its specific surface area.

\section{Image processing.}

Images are processed by computing and analyzing the shape of the gray level contours (GL) generated by the droplets in the volume visited for each tomographic shot ${ }^{23}$. Images are encoded by the camera with 256 gray levels running from 0 (black) to 255 (white) and denoted in the following by the integer $\mathrm{x}_{\mathrm{GL}}$ (with $\mathrm{x}_{\mathrm{GL}}=0,1, \ldots 255$ ). In order to limit data handling, only 32 equidistant gray levels are used in this study $\left(\mathrm{x}_{\mathrm{GL}}=0,8,16, \ldots, 248\right)$. Droplets are hence described by a maximum of 32 contours that actually reflect their morphological characteristics. Spherical droplets will predominantly give rise to circular contours whereas polymorphous ones will yield more complex geometries (see magnified droplets in the insets Figure 1). (a)

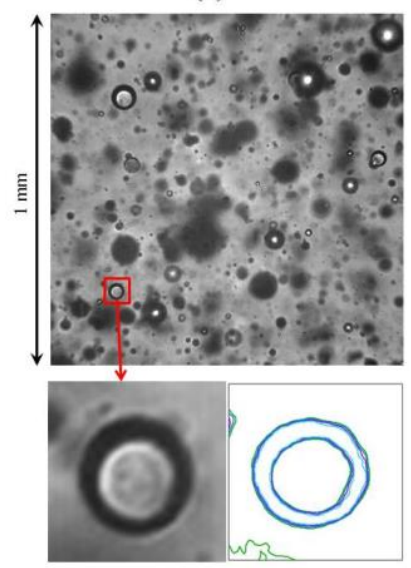

(b)

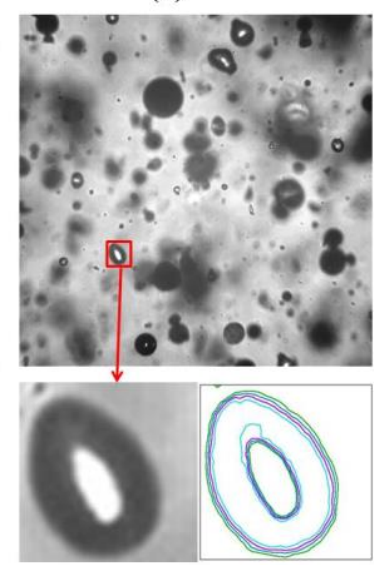

Figure 1: Picture of an emulsion with spherical droplet (a) and polymorphous ones (b). $\left[\mathrm{SiO}_{2}\right]=0 \mathrm{~g} / \mathrm{L}$ for (a) while $\left[\mathrm{SiO}_{2}\right]=270 \mathrm{~g} / \mathrm{L}$ for (b). For both emulsions, $[\mathrm{CTAB}]=0,365 \mathrm{~g} / \mathrm{L}$. The composition of the SMN in (b) corresponds to SMN P5 in Table 3. Two droplets in focus with comparable sizes are magnified (bottom images) and corresponding gray level contours displayed. The field of view is $1 \mathrm{~mm}^{2}$.

The degree of deformation of a droplet is estimated, for a given value of $\mathrm{x}_{\mathrm{GL}}$, by the standard deviation $\mathrm{x}_{\sigma}$ :

$$
x_{\sigma}=\sqrt{\frac{1}{N_{\text {cont }}} \sum_{i=1}^{N_{\text {cont }}}\left(r_{i}-\langle r\rangle\right)^{2}}
$$

with

$$
r_{i}=\sqrt{\left(x_{i}-x_{c e n t}\right)^{2}+\left(y_{i}-y_{\text {cent }}\right)^{2}}
$$

and

$$
x_{\text {cent }}=\frac{1}{N_{\text {cont }}} \sum_{i=1}^{N_{\text {cont }}} x_{i} \quad, \quad y_{\text {cent }}=\frac{1}{N_{\text {cont }}} \sum_{i=1}^{N_{\text {cont }}} y_{i}
$$

$\left(x_{i}, y_{i}\right)$ is the position of the $i^{\text {th }}$ node of the considered GL contour, $\mathrm{N}_{\text {cont }}$ is total the number of nodes of this contour and $\left(\mathrm{x}_{\text {cent }}, \mathrm{y}_{\text {cent }}\right)$ the position of its barycenter (see Figure 2). The distance between each contour node and its barycenter is given by $r_{i}$ and $\langle r\rangle$ is the radius of the circle with the same perimeter than the considered contour. Variable $x_{\sigma}$ gives a quantitative estimation of the distortion level of the contours for a given $\mathrm{x}_{\mathrm{GL}}$. If $\mathrm{x}_{\sigma}=0$, contours are circles whereas if $x_{\sigma} \neq 0$, they deviate from a circular geometry. But considering only one value of $\mathrm{x}_{\mathrm{G}}$ is not sufficient for the purpose of this study. Aggregated droplets can for example give rise to deformed contours even though droplets are spherical. The previous procedure is therefore iterated for all the 32 values of $\mathrm{x}_{\mathrm{GL}}$ and for all the tomographic images where the considered droplet can be identified. For the droplets displayed in the insets of Figure 1, such an analysis generates 20 contours in average and up to 32 contours for well contrasted droplets like the one in the inset of Figure 1(b). When performing tomographic shots, small droplets remain well contrasted over a distance that is close to their radius. Each droplet therefore produces a large number of contours. This number grows with the droplets radius because, when larger, they become better contrasted due to the small depth of field. For the droplet in the inset of Figure 1(b), the tomographic procedure will produce more than 400 contours. This is far enough to conclude about its shape. 100 contours are indeed already sufficient for statistical relevance. For small droplets however, this approach does not hold. The number of points for each given GL is indeed too small. A $5 \mu \mathrm{m}$ cut off in the diameter of the droplets is therefore introduced. Below this limit the droplets will not be accounted for in the analysis.
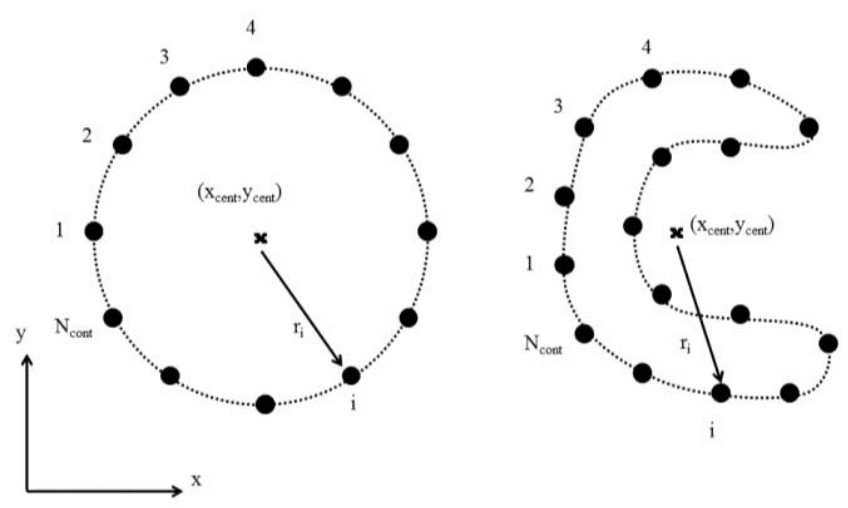

Figure 2: Schematic view of the contouring technique and illustration of the variables of Eq. 1 to Eq. 3. For a circular contour (left) and for a deformed one (right). The numbers correspond to the index of the nodes and ( $\mathrm{x}_{\text {cent }}$, $\mathrm{y}_{\text {cent }}$ ) to the position of the barycenter of the contours.

All the emulsions that will be investigated exhibit at least 100 droplet $/ \mathrm{mm}^{3}$ with diameter larger than $5 \mu \mathrm{m}$ and the minimal number of contours to be treated is several ten thousands. It reaches more than $10^{7}$ for highly dispersed and well contrasted emulsions. As just discussed, this large number of data makes reasonable a statistic treatment. When running over all values of $\mathrm{x}_{\mathrm{GL}}$ and $\mathrm{x}_{\sigma}$, it is possible to generate a distribution function denoted $\mathrm{f}_{\mathrm{raw}}\left(\mathrm{x}_{\mathrm{GL}}, \mathrm{x}_{\sigma}\right)$ that maps the structure of the emulsion into a $3 \mathrm{D}$ surface as illustrated in Figure 3(a) for the emulsion of Figure 1(b). For 
a given value of $\mathrm{x}_{\mathrm{GL}}$, this function always shows a decrease of $\mathrm{f}_{\text {raw }}$ when $\mathrm{x}_{\sigma}$ increases. Ideally, this decay should not show up for emulsions with spherical droplets like in Figure 3(a) since all contours should be circles. Distortion of these latter is actually a consequence of the foreground and background fields where droplets are not in focus. Despite this undesirable effect, the overall distortion of the contours is always smaller for spherical droplet emulsions (SDE) than for polymorphous droplets emulsion (PDE). This trend will help to discriminate SDE from PDE.

Several strategies can be followed at this point. The most straightforward is to directly use the decreasing trend of $\mathrm{f}_{\text {raw }}$ with $\mathrm{x}_{\sigma}$. If it is fast (resp. slow), the proportion of circular (resp. non circular) contours will be larger (resp. smaller). In the first case droplets will be predominantly spherical and emulsions SDEs while in the second, droplets will be polymorphous and emulsions will correspond to PDEs. The shape of $f_{\text {raw }}$ is described using the fitting function $\mathrm{f}_{\mathrm{fit}}\left(\mathrm{x}_{\mathrm{GL}}, \mathrm{x}_{\sigma}\right)$ defined by :

$$
f_{f i t}\left(x_{G L}, x_{\sigma}\right)=a \times \exp \left(-\frac{\left(x_{G L}-b\right)^{2}}{c}\right) \times \exp \left(-\xi \times x_{\sigma}\right)
$$

where $\mathrm{a}, \mathrm{b}, \mathrm{c}$ and $\xi$ are positive fitting parameters obtained from a non-linear least square fitting. This expression of $f_{\text {fit }}$ is motivated by the shape of $f_{\text {raw }}$ (Figure 3(a)). For given $x_{\sigma}$, it exhibits a peaked shape that declines rapidly and tends to flatten for both large and small values of $\mathrm{x}_{\mathrm{GL}}$. Assuming a Gaussian shaped function for $\mathrm{x}_{\mathrm{GL}}$ is therefore reasonable. Other functions like, for example, Lorentzian curves could also have been used here. But fitting would not be significantly improved. For the $\mathrm{x}_{\sigma}$ dependency, an exponential decay is assumed by analogy with what is done in statistical analysis when considering correlation functions. The difference between SDEs of PDEs indeed relies on how similar the contours are (for a given value of $\mathrm{x}_{\mathrm{GL}}$ ) and therefore how fast statistical correlation function vanishes. This trend is given by $\xi$ in Eq. 4 .

The ability of this parameter to properly catch the structural properties of two different emulsions actually also depends on the quality of the treated images and in particular on their contrast. Special care has been devoted to this problem. As emulsions are studied in transmitted light mode, high dispersion (i.e. small droplets) can seriously degrade image quality due to light scattering and opacity. One straightforward procedure to tackle with this problem is to keep the reference focus position of the microscope fixed, to adjust contrasts by optimizing optical parameters (LED intensity, exposure time, iris opening, etc) and to control image quality with a direct visual inspection before starting the scanning shots further inside the emulsion. The advantage of such an approach is to optimize grey level contouring and therefore maximizing the number of contours to be treated while keeping the focus of the microscope far inside the emulsion. But this is not always possible due to transparency constraints. It has moreover the drawback to require the monitoring of many optical parameters that are not easy to tune independently and that depend strongly on the properties of the emulsions. This is why another strategy is followed in this work. It consists to keep all optical parameters unchanged and to only move the focus of the microscope to a reference location where contrast is sufficient for precise droplet identification. Exposure time is set to $1 / 500 \mathrm{~s}$ and LED intensity is maximal. Tests on the most opaque PDE that will be considered (see Figure 7(f)) indicate that iris opening set to
$30 \%$ is a reasonable compromise as long as emulsions show more than 500 droplets per $\mathrm{mm}^{3}$ (Figure 7(b)). With this value, the diameter of the incident light beam is close to 0.9 $\mathrm{cm}$. For smaller amounts of droplets, iris opening has been slightly reduced to avoid saturation of the CCD camera. This is the case only for the emulsion of Figure 7(a). This has no significant incidence on the overall shape of $f_{\text {raw }}$ since it is only the number of contours to be treated that is modified and not their geometry. In summary, for the strategy followed here, the essential parameter for contrast quality is the initial position of the focus of the microscope. It is moved from the quartz cell center, for transparent emulsions, to the vicinity of the front window, for opaque ones. Minimal distance between focus and front window is $0.5 \mathrm{~mm}$ to get rid of wall effects. With all the previous settings, $\xi$ ranges between 2.5 for SDEs and 1.5 for PDEs and $\xi=2$ will be considered as the criterion for PDE/SDE discrimination.
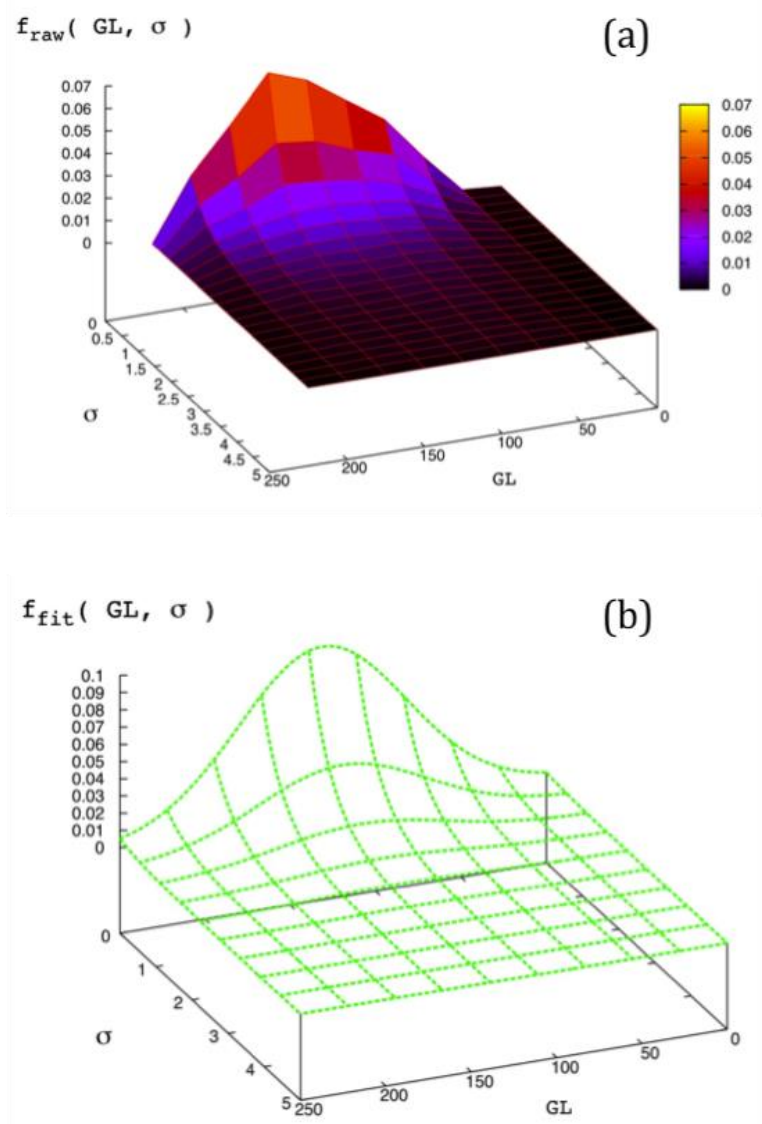

Figure 3 : (a) raw distribution $\mathrm{f}_{\mathrm{raw}}\left(\mathrm{x}_{\mathrm{GL}}, \mathrm{x}_{\sigma}\right)$ as a function of the gray level $\left(\mathrm{x}_{\mathrm{GL}}\right)$ and contour deformation $\left(\mathrm{x}_{\sigma}\right)$ for a single scanning shot of the emulsion of Figure 1(b). (b) fitting function $\mathrm{f}_{\text {fit }}$ of (a).

\section{Results and discussion}

The emulsions studied hereafter are emulsions characterized by a dispersed phase consisting of a liquid suspension of nanoparticles. Conversely to RamsdenPickering emulsions where solid particles are usually in the continuous phase here it is the dispersed phase that contains these latter. This means that bulk nanoparticles microstructures, if any, are located inside the droplets or at the water/PO interface and not in the continuous phase. When $[\mathrm{SPAN} 80]=0 \mathrm{~g} / \mathrm{L}$ it has been shown that the balance between $\mathrm{CTAB}$ and $\mathrm{SiO}_{2}$ concentrations determines a 
critical mixing ratio $\mathrm{R}_{\mathrm{c}} \approx 0.03$ where the transition from SDE to PDE occurs ${ }^{23-25}$.

\subsection{General structure of the emulsions.}

Figure 4 gives a first illustration of what happens when adding increasing amounts of SPAN80 into the PO phase of such a sytem. It represents images of six emulsions denoted S1 to S6. Their composition is given in Table 1. They're all such that $\mathrm{R}<\mathrm{R}_{\mathrm{c}}$ and therefore all have the structure of a PDE when SPAN80 free (see also Figure 1(b)). The left column of Figure 4 shows emulsions with [SPAN80] $=0 \mathrm{~g} / \mathrm{L}(\mathrm{S} 1, \mathrm{~S} 3, \mathrm{~S} 5)$ that all correspond to PDEs. The right one corresponds to emulsions with [SPAN80] $=4.2510^{-2} \mathrm{~g} / \mathrm{L}$ (S2, S4, S6). Surprisingly, these latter all exhibit spherical droplets and are therefore SDEs. This is clearly evidenced in the enlargements of each image and demonstrates that the addition of a small amount of SPAN80 generates important structural modifications. The qualitative description of this phenomenon is actually the main focus of this work. It is also important to note at this point that, for the concentration ranges treated in Table 1, the transition from PDE to SDE does not depend strongly on the concentration of $\mathrm{SiO}_{2}$ and CTAB. This can be qualitatively checked by comparing $(\mathrm{S} 1, \mathrm{~S} 3)$ and $(\mathrm{S} 2, \mathrm{~S} 4)$ (resp. (S3, S5) and (S4, S6)) where $\left[\mathrm{SiO}_{2}\right]$ (resp. [CTAB]) is increased by one order of magnitude. No significant structural changes of the emulsions are observed as the typical shape of the droplets (spherical or polymorphous) is unchanged. This will be addressed qualitatively hereafter. Higher emulsification is here a straightforward consequence of larger surfactant concentrations.
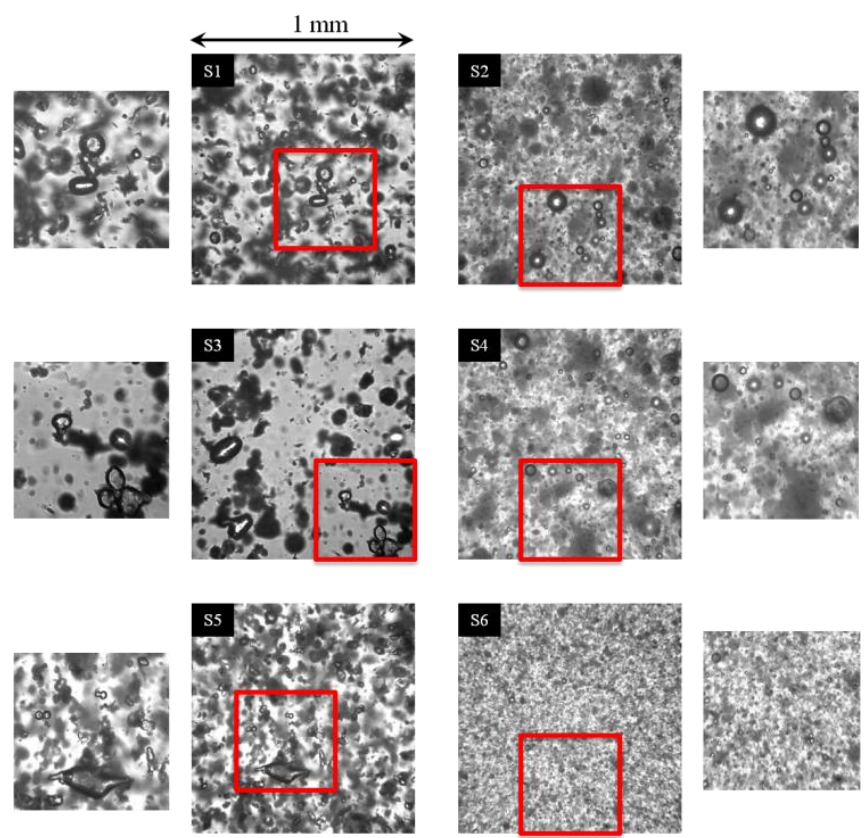

Figure 4 : Images of emulsions for compositions given in Table 1. For S1, $\mathrm{S} 3, \mathrm{~S} 5$ [SPAN80] $=0 \mathrm{~g} / \mathrm{L}$ and for S2, S4, S6 [SPAN80] $=4.2510^{-2} \mathrm{~g} / \mathrm{L}$. Left and right pictures show enlargements of the square of each image.

The above observations can be reproduced for SDS/alumina nanoparticles as illustrated in Figure 5 for the compositions of Table 2 where emulsions are all PDEs when SPAN80 free ${ }^{34}$. Again, the transition from PDE to SDE depends on the SPAN80 concentration. When [SPAN80]=0 $\mathrm{g} / \mathrm{L}$ (S1', S3' and S5') droplets become smaller due to modified SDS and alumina nanoparticles concentrations, dispersion is improved but no structural changes occur in the droplets shape. This is no more true when SPAN80 is added as clearly demonstrated when comparing (S1', S2'), (S3', $\mathrm{S}^{\prime}$ ') or (S5', S6'). Similarly to $\mathrm{CTAB} / \mathrm{SiO}_{2}$ emulsions, SDS/Alumina ones have to be transparent for OTM investigations and moreover to be PDEs before addition of SPAN80. This is the reason why the range of concentrations used for SDS (resp. alumina nanoparticles) is not as broad as for CTAB (resp. $\mathrm{SiO}_{2}$ nanoparticles) (see Table 1 and 2). Changing them by one order of magnitude as done for the $\mathrm{CTAB} / \mathrm{SiO}_{2} \mathrm{SMNs}$ would lead to opaque emulsions and therefore make the OTM approach no more suitable for our purpose. This is the reason why focus will be put from now up only on $\mathrm{CTAB} / \mathrm{SiO}_{2} \mathrm{SMNs}$.
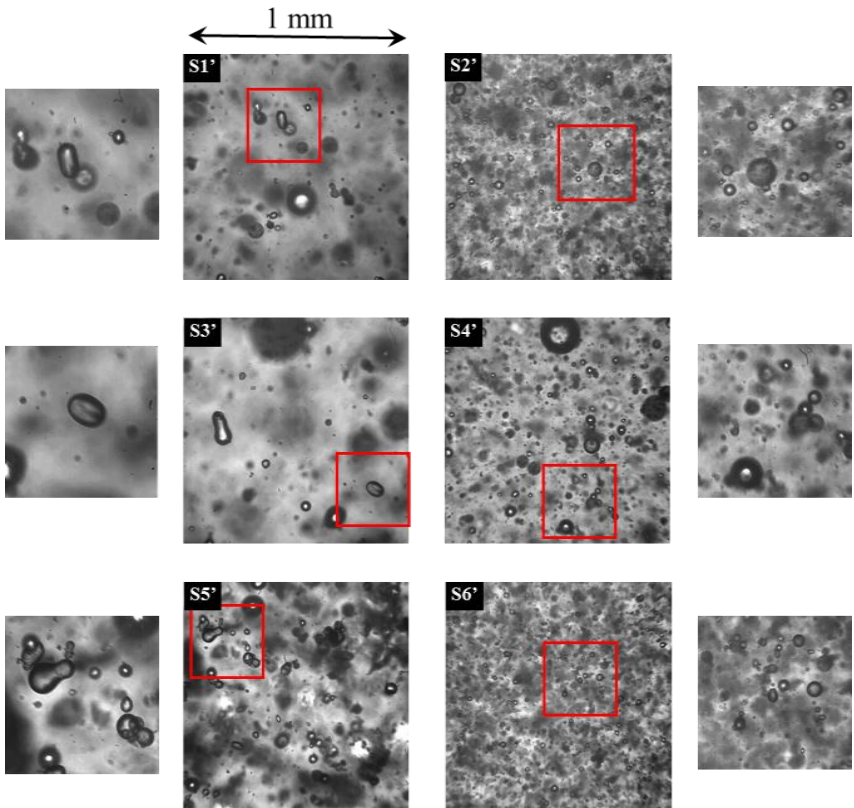

Figure 5: Same than in Figure 4 but for SDS/Alumina SMNs with composition given in Table 2. For S1', S3', S5' [SPAN80] =0 g/L and for S2', S4', S6' [SPAN80]= $0.1 \mathrm{~g} / \mathrm{L}$

Figure 6 illustrates in a 2D plot, the different compositions of the $\mathrm{CTAB} / \mathrm{SiO}_{2} \mathrm{SMNs}$ that will be studied. $\mathrm{SiO}_{2}$ concentration runs from $1 \mathrm{~g} / \mathrm{L}$ up to $300 \mathrm{~g} / \mathrm{L}$ while the CTAB one, is between $10^{-3}$ and $10 \mathrm{~g} / \mathrm{L}$. All these solutions have been obtained from dilutions of the same stock solution. This explains why both CTAB and $\mathrm{SiO}_{2}$ concentration points change from one solution to the other. Figure 6 indicates that three distinct composition domains can be identified ${ }^{25}$. In the first one (D1), emulsions are SDEs (Figure 1(a)). It is such that $[$ CTAB] $>0.03 \mathrm{~g} / \mathrm{L}$ and $[\mathrm{CTAB}] /\left[\mathrm{SiO}_{2}\right]>0.03$. In the second one (D2) $[\mathrm{CTAB}]>0.03 \mathrm{~g} / \mathrm{L}$ and $[\mathrm{CTAB}] /\left[\mathrm{SiO}_{2}\right]<0.03$. Emulsification is still possible but emulsions are now PDEs (Figure 1(b)). When $[\mathrm{CTAB}]<0.03 \mathrm{~g} / \mathrm{L}$ (D3) emulsification is no more possible with the protocol used in this work. This is just a straightforward consequence of the absence of surface active properties of $\left[\mathrm{SiO}_{2}\right]$. 


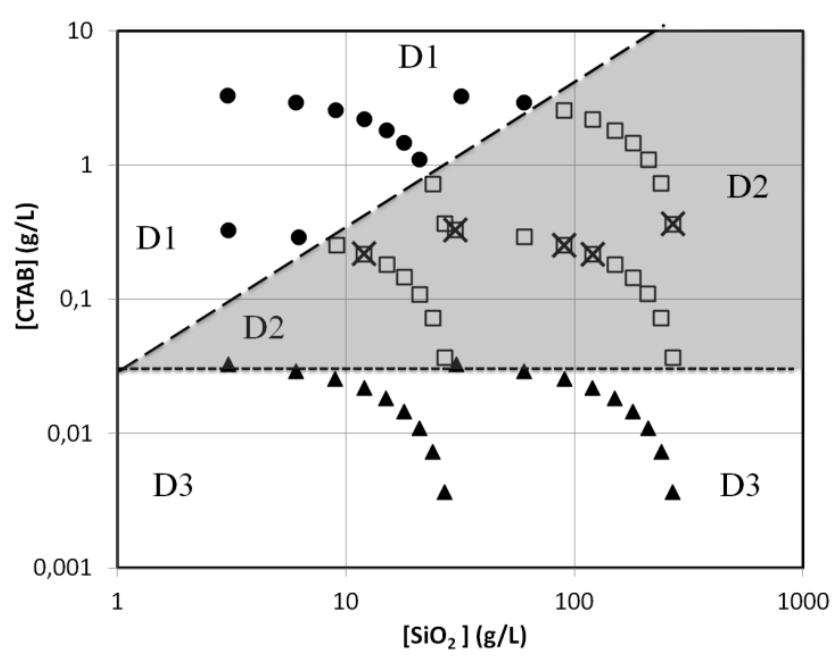

Figure 6: Nanofluid composition and emulsion structure as a function of $[\mathrm{CTAB}]$ and $\left[\mathrm{SiO}_{2}\right]$ for $[\mathrm{SPAN80}]=0 \mathrm{~g} / \mathrm{L} . \mathrm{D} 1, \mathrm{D} 2$ and D3 are three specific composition domains. Markers $\bullet, \square$ and $\boldsymbol{\Delta}$ correspond to compositions points respectively in D1, D2 and D3. The gray domain emphasizes SMNs giving rise to PDEs and the five crosses correspond to the SMN compositions given in Table 3. The long dashed line is the SDE/PDE transition region $\left(\mathrm{R}=\mathrm{R}_{\mathrm{c}}\right)$ and the short dashed line is the CTAB concentration limit below which emulsification is no more possible.

In Figure 6, the five crosses correspond to the five SMNs that will be studied next to illustrate what happens for increasing concentrations of SPAN80. They're denoted by $\mathrm{P} 1$ to $\mathrm{P} 5$ and their composition is given in Table 3. [CTAB] is kept in the same range while $\left[\mathrm{SiO}_{2}\right]$ spans over one order of magnitude. Other compositions could be studied here in particular with better controlled CTAB concentrations similarly to what was done for the emulsions of Table 1 (see Figure 4). But this would just make SMN preparation more complex without any significant changes in the final results. Important is to note here that all the SMNs are in D2 and therefore give rise to PDEs when $[S P A N 80]=0 \mathrm{~g} / \mathrm{L}$.

\begin{tabular}{|c|c|c|c|}
\hline Name & {$[\mathrm{CTAB}](\mathrm{g} / \mathrm{L})$} & {$\left[\mathrm{SiO}_{2}\right](\mathrm{g} / \mathrm{L})$} & $\mathrm{R}$ \\
\hline $\mathrm{P} 1$ & 0,219 & 12 & 0.018 \\
\hline $\mathrm{P} 2$ & 0,328 & 30 & 0.011 \\
\hline P3 & 0,255 & 90 & 0.003 \\
\hline P4 & 0,218 & 120 & 0.002 \\
\hline P5 & 0,365 & 270 & 0.001 \\
\hline
\end{tabular}

Table 3: Composition of the five SMNs (crosses in Figure 6). The values of $\mathrm{R}$ are also presented and are such that $\mathrm{R}<0.03$ as expected for PDEs.

SPAN80 is hydrophobic (HLB = 4.3) and mostly remains in $\mathrm{PO}$ or adsorbs on $\mathrm{PO} / \mathrm{SMN}$ interfaces whereas $\mathrm{CTAB}$ is hydrophilic $(\mathrm{HLB}=10)$ and therefore remains preferentially in the SMN or adsorbs on the PO/SMN interface. The structural modifications illustrated in Figure 4 when adding SPAN80 therefore raise several fundamental questions: (i) what is the effect of the addition SPAN80 on the PO/SMN interfacial properties ? (ii) is SPAN80 interacting with the $\mathrm{SiO}_{2}$ nanoparticles when they become partially hydrophobic due to increasing CTAB concentrations ? (iii) what about possible micellar transport of the SMN into PO and vice versa?

\subsection{Effect of the addition of SPAN80.}

The effect of the addition of SPAN80 on the structure of emulsion $\mathrm{P} 1$ will be considered first. It is illustrated in Figure 7 where [SPAN80] is progressively increased from $0 \mathrm{~g} / \mathrm{L}$ (a) to $4.2510^{-2} \mathrm{~g} / \mathrm{L}$ (f). When polymorphous, droplets behave similarly to the ones discussed in Figure 1(b) and Figure 4 (left column). Their shape is indeed unaffected by the overall hydrodynamics of the emulsion and coalescence hindered. This stiffness is a consequence of the self-organization of the silica nanoparticles inside droplets and/or on their interface ${ }^{24,25,30}$. Self-organized structures are known to show up only when CTAB is added into the SMN and have been shown to create visco-elastic behaviors but with relatively small elastic moduli ${ }^{28}$. They consist in weak microstructures that easily reorganize under small external constrains. The precise contribution of bulk and interfacial phenomena to explain droplet deformation and transition from PDE to SDE is still in discussion. Bulk properties seem indeed not sufficient to fully explain this phenomenon.

Figure 7(a) shows a PDE with droplets having sizes larger than $50 \mu \mathrm{m}$, most of which are polymorphous. When increasing [SPAN80] the emulsification is improved, dispersion increased and the typical size of the droplets decreased as can be seen in Figure 7(b) to (f). As the emulsification procedure is the same for all the samples, it is obvious here that the improved dispersion of the SMN is a consequence of the smaller surface tension resulting from the addition of SPAN80. It indeed comes in addition to CTAB and CTAB-modified $\mathrm{SiO}_{2}$ nanoparticles. The production of emulsions with an improved stability when adding a surfactant is therefore clearly expected here. It simply results from synergistic effects between two surfactants. But the images of Figure 7 also demonstrate that SPAN80 can be used to tune the emulsion structure from PDE (images $a, b$ and c) to SDE (images $d$, e and f). The recovery of spherically shaped droplets when increasing SPAN80 concentrations is surprizing in particular when keeping in mind that emulsification procedure and volume fractions are exactly the same.

To our knowledge, the precise mechanism(s) explaining this phenomenon is(are) not yet clearly identified. One possibility is the occurrence of larger mechanical constraints due to increasing capillary pressure that becomes strong enough to overcome microstructure stiffness. Elastic modulus of the microstructures is indeed small ${ }^{28}$, weak changes in the external constrains will hence bend them and therefore make their arrangement fit with the spherical geometry imposed to droplets by larger capillary pressure. Such a scenario clearly holds when the addition of SPAN80 drastically changes the emulsion dispersion as observed for S5 and S6 in Figure 4. For smaller concentrations however, many large spherical droplets remain as can be seen when comparing S1 (resp. S3) and S2 (resp. S4). This indicates that adsorption/desorption processes are probably also involved. Simultaneously to stiffness modifications, drastic changes can indeed occur in the adsorption properties of the $\mathrm{SiO}_{2}$ microstructures for large SPAN80 concentrations. These hypotheses and mechanisms will be discussed in more details below in the last part of this article. 

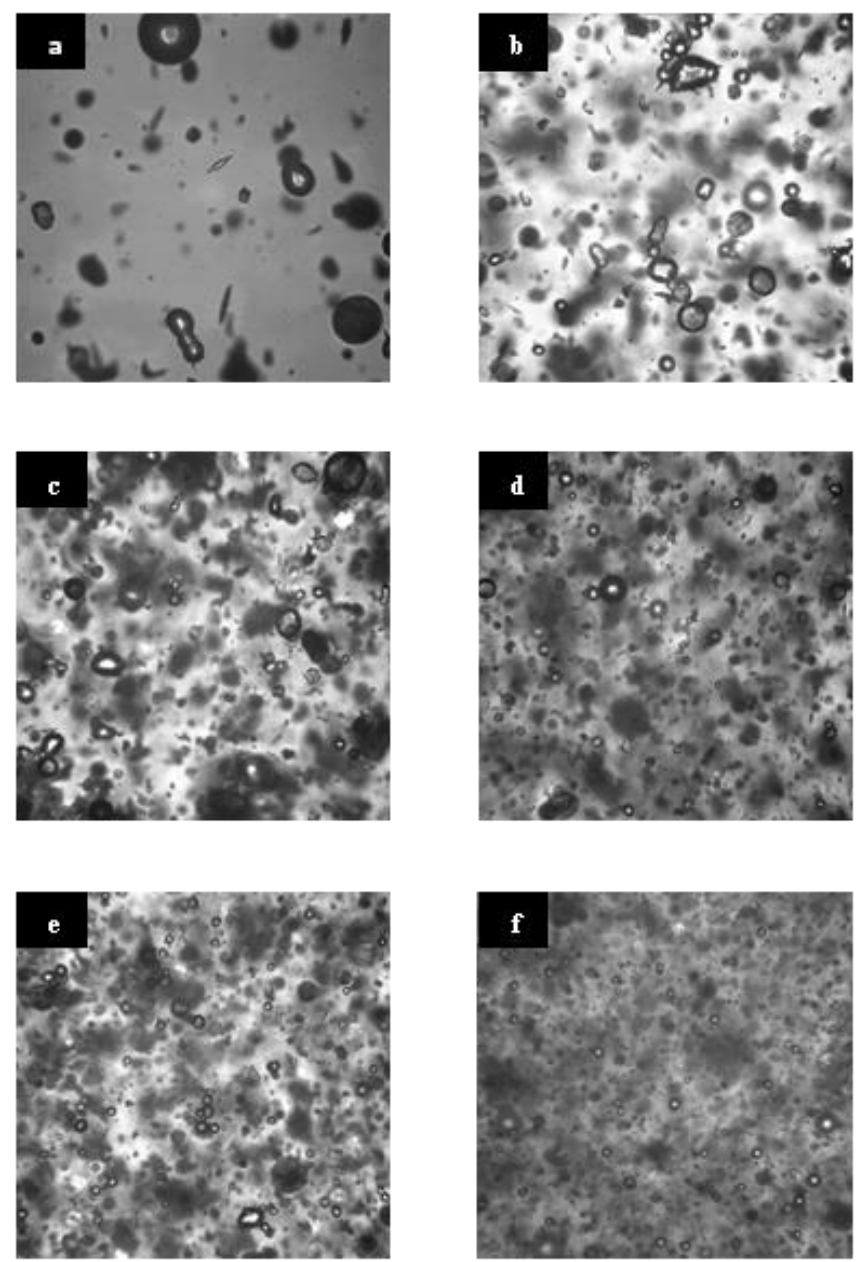

Figure 7: Images of emulsion P1 with increasing SPAN80 concentration from (a) to (f). (a) $\left[\right.$ SPAN80] $=0 \mathrm{~g} / \mathrm{L}$, (b) $\left[\right.$ SPAN80] $=5.0010^{-3} \mathrm{~g} / \mathrm{L}$, (c) [SPAN80] $=1.5010^{-2}$, (d) [SPAN80] $=2.5010^{-2} \mathrm{~g} / \mathrm{L}$, (e) [SPAN80] $=3.5010^{-}$ ${ }^{2} \mathrm{~g} / \mathrm{L}$, (f) $[\mathrm{SPAN} 80]=4.2510^{-2} \mathrm{~g} / \mathrm{L}$. The field of view is the same than in Figure 1 and Figure 4.

The experiments of Figure 7 have been reproduced for all the SMNs of Table 3. The observed phenomenology is always the same than the one just discussed. The idea is now to give a quantitative criterion to estimate the SPAN80 concentration for which the transition from PDE to SDE occurs. To this end, three consecutive scanning shots separated by 5 minutes periods of time are performed for each studied emulsion. This will help to improve reliability of the measurements and to account for possible time drifts generated by emulsion drainage. Figure 8 displays the resulting values of $\xi$ (see Eq. 4) as a function of [SPAN80]. The filled circles represent the average value of $\xi$, denoted $\langle\xi\rangle$, of all the measurements for given [SPAN80]. $\xi<2$ as long as [SPAN80] $<0.02 \mathrm{~g} / \mathrm{L}$ while for [SPAN80] $>0.06 \mathrm{~g} / \mathrm{L}$, $\xi>2$. This is the signature of the change in the emulsion structure from PDE to SDE. For $0.02 \mathrm{~g} / \mathrm{L}<[$ SPAN80] $<0.06$ $\mathrm{g} / \mathrm{L}$, the values of $\xi$ are spread indicating that the contributions of spherical and deformed droplets have a similar weight. The transition composition is denoted by $[\mathrm{SPAN} 80]_{\mathrm{c}}$ and corresponds to the value of [SPAN80] for which $\langle\xi\rangle=2$. It is obtained from an hyperbolic fitting of $<\xi>$ and gives $[\text { SPAN80 }]_{\mathrm{c}}=0.035 \pm 0.005 \mathrm{~g} / \mathrm{L}$.

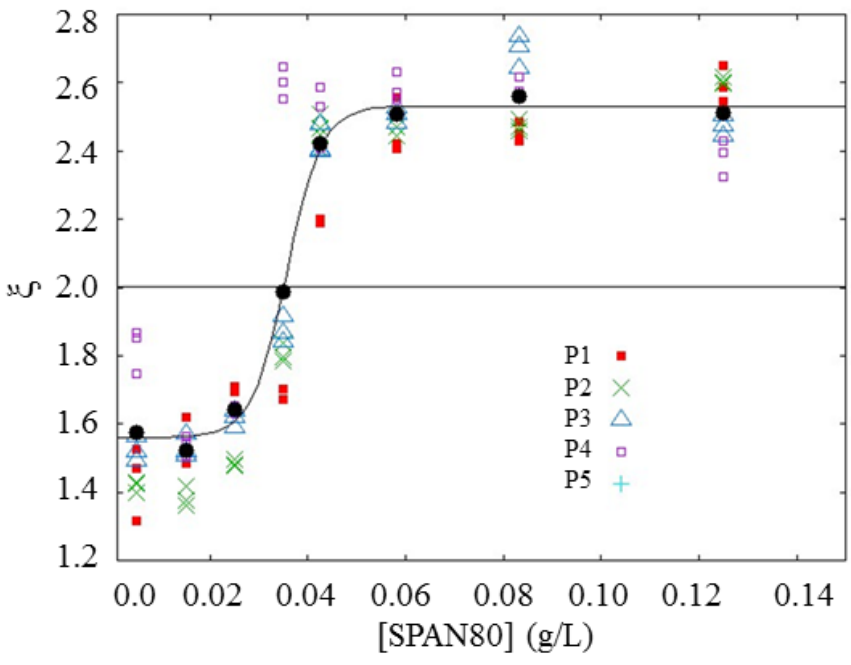

Figure $8: \xi$ as a function of [SPAN80] when emulsifying SMNs P1 to P5 (Table 3). Filled circles represent the average value $\langle\xi\rangle$ for given [SPAN80]. The full line is an hyperbolic tangent fitting. The transition region from PDEs to SDEs occurs when when $\langle\xi\rangle=2$ where [SPAN80]= $0.035 \pm 0.005 \mathrm{~g} / \mathrm{L}$

The concentration ranges of SPAN80 have been limited to [SPAN80] $<0.14 \mathrm{~g} / \mathrm{L}$. For larger values, emulsions are too opaque to be operated with OTM. This opaqueness is due to the stabilization of microdroplets that cannot be captured in the present working conditions. Optical resolution is indeed limited to $1 \mu \mathrm{m}$ and imaging technique holds only for droplets with diameter larger than $5 \mu \mathrm{m}$. As a result, microdroplets are accounted neither for the distributions of Figure 3 nor for the value of $\langle\xi\rangle$. The imaging technique used here is not adapted for highly dispersed emulsions. Reflection microscopy could be used. But low contrast levels will also be limiting in that case. This technique has moreover the important drawback to restrict emulsion investigations to narrow domains (few tens of microns) in the vicinity of the windows of the containers where uncontrolled wall effects might occur. The SPAN80 concentration range of Figure 8 is actually sufficient for the construction of a relevant composition diagram where all chemical compounds are accounted for. Figure 9 gives an illustration of such a diagram in a 3D representation. The compositions of the SMNs are the ones in D2 (Figure 6) and up to 10 different SPAN80 concentrations have been used for each SMN. PDEs (resp. SDEs) are represented with crosses (resp. filled squares). This figure indicates that the transition between PDE and SDE occurs for [SPAN80] $\approx 0.03$ $\mathrm{g} / \mathrm{L}$ for all the considered values of $\left[\mathrm{SiO}_{2}\right]$ when $[\mathrm{CTAB}]<0.2 \mathrm{~g} / \mathrm{L}$. Surprisingly, SPAN80 is the only driving chemical compound regarding to emulsion structure when $[\mathrm{CTAB}]<0.2 \mathrm{~g} / \mathrm{L}$. Nanoparticles act therefore as buffers for CTAB. This is evidenced by the flat domain of Figure 9. For larger values of [CTAB] however, the transition threshold increases and becomes [CTAB]-dependent. 


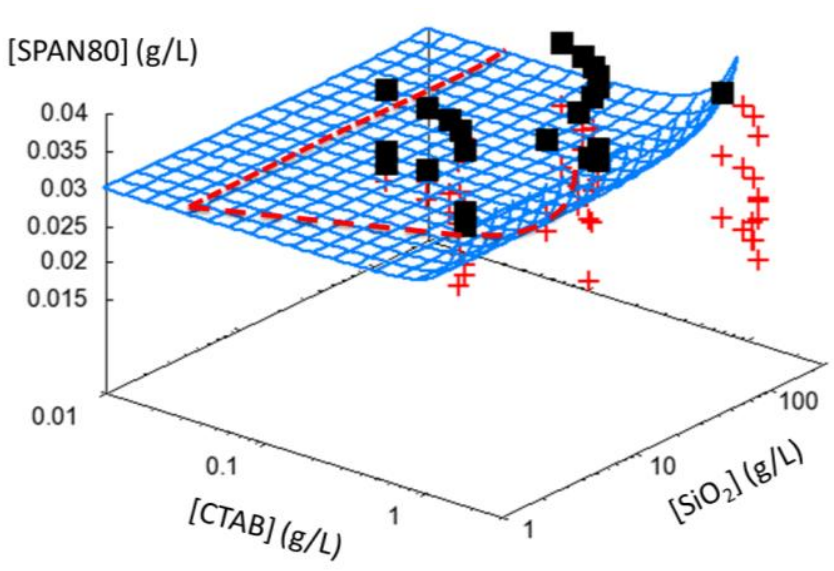

Figure 9: Transition region between $\mathrm{PDE}$ and $\mathrm{SDE}$ as a function of [CTAB], $\left[\mathrm{SiO}_{2}\right]$ and [SPAN80]. Crosses (resp. squares) correspond to PDE (resp. SDE). A surface fitting of the PDE/SDE transition region is shown to guide the eyes. The dashed lines are the limits of D2 (Figure 6).

\subsection{Air-CTAB/SiO $2 \mathrm{SMN}$ interfacial properties.}

At this stage, it is important to focus a moment on the properties of the $\mathrm{CTAB} / \mathrm{SiO}_{2} \mathrm{SMNs}$ under consideration here and in particular on the partition properties of CTAB. Figure 10 is a $3 \mathrm{D}$ plot of the behaviour of the interfacial tension $\sigma$ of $\mathrm{CTAB} / \mathrm{SiO}_{2}$-Air interfaces at $20^{\circ} \mathrm{C}$. Measurements are performed with the Du Nouÿ technique. For these experiments, [CTAB] is controlled in the SMN preparation but not $\left[\mathrm{SiO}_{2}\right]$. This is why $\left[\mathrm{SiO}_{2}\right]$ again varies for each $\mathrm{CTAB}$ increment. The relevance of SMNs-Air properties in the context of this work will be discussed in more detail below. Figure 10 shows that $\sigma \approx 70 \mathrm{mN} / \mathrm{m}$ when $[\mathrm{CTAB}]<0.2 \mathrm{~g} / \mathrm{L}$ for all values of $\left[\mathrm{SiO}_{2}\right]$. This is close to the one of pure water and reveals the high affinity of CTAB monomers with the $\mathrm{SiO}_{2}$ nanoparticles ${ }^{31,36}$. The fall of $\sigma$ for larger values demonstrates that for moderated $\mathrm{SiO}_{2}$ concentrations $\left(\left[\mathrm{SiO}_{2}\right]<0.1 \mathrm{~g} / \mathrm{L}\right), \mathrm{CMC}$ of CTAB remains close to $0.3 \mathrm{~g} / \mathrm{L}$ as expected from literature for pure water. It also indicates that when increasing the loading of the SMNs in nanoparticles, $\sigma$ still drops but for larger CTAB concentrations. This confirms again that CTAB preferentially adsorbs on $\mathrm{SiO}_{2}$ nanoparticles and that there are only residual amounts of free surfactant in the continuous aqueous phase of the SMNs when $R<R_{c}$. When [CTAB] exceeds CMC, nanoparticle coverage becomes complex as multi-layers are generated. Silica nanoparticles can then aggregate due to neutralization of zeta potential and $\mathrm{SiO}_{2}$ microstructures start to form ${ }^{36}$. The composition of the SMNs for which Figure 10 indicates a clear fall of $\sigma$ follows a line along which the mixing ratio is constant with $\mathrm{R}=\mathrm{R}_{\mathrm{c}} \approx 0.03$. The plot of $\sigma$ as a function of $\mathrm{R}$ is represented in Figure 11. The measurement points nicely overlap and $\sigma$ drops down to $35 \mathrm{mN} / \mathrm{m}$ at $\mathrm{R}=\mathrm{R}_{\mathrm{c}}$.

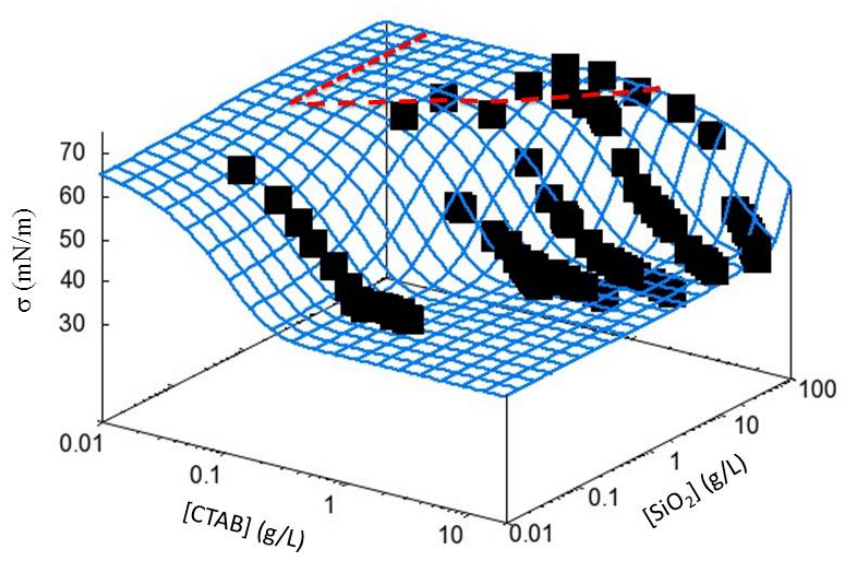

Figure 10: 3D plot of the interfacial tension of SMS-air interfaces as a function of $[\mathrm{CTAB}]$ and $\left[\mathrm{SiO}_{2}\right]$. The surface is presented to guide eyes. The dashed lines are the limits of D2 (Figure 6).

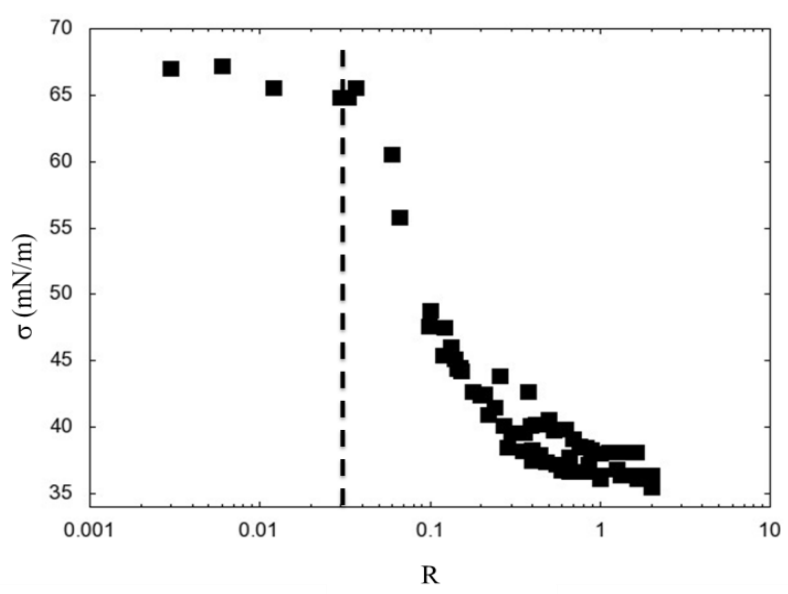

Figure 11: Interfacial tension as a function of the mixing ratio $[\mathrm{CTAB}] /\left[\mathrm{SiO}_{2}\right]$ for the measurement points of Figure 10. The vertical line corresponds to $\mathrm{R}=\mathrm{R}_{\mathrm{c}} \approx 0.03$.

Figure 12 is a plot of the Gibbs adsorption isotherm $\Gamma$ computed from all the data of Figure $10 . \Gamma$ is the excess molar surface concentration and $[\mathrm{CTAB}]_{\mathrm{eq}}$ is the corresponding equilibrium value of the $\mathrm{CTAB}$ concentration in the continuous aqueous phase when $\left[\mathrm{SiO}_{2}\right]=0 \mathrm{~g} / \mathrm{L}$. A sigmoid shape is obtained with an inflexion point at $[\mathrm{CTAB}]_{\mathrm{eq}} \approx 0,15 \mathrm{~g} / \mathrm{L}$. It indicates that CTAB monolayers are formed on the nanoparticles and that room is still available for new adsorbing CTAB molecules as long as $[\mathrm{CTAB}]_{\mathrm{eq}}<0,15 \mathrm{~g} / \mathrm{L}$. The saturation concentration $[\mathrm{CTAB}]_{\mathrm{s}}$ is defined, for a given value of $\left[\mathrm{SiO}_{2}\right]$, as the maximal CTAB concentration in the SMNs beyond which multilayers start to be generated. According to what was just discussed $[\mathrm{CTAB}]_{\mathrm{s}} \approx 0,15 \mathrm{~g} / \mathrm{L}$. For larger values, adsorption layers have more complex structures and can show up as multilayers, hemi-micelles or coarse CTAB aggregates. When approaching $\mathrm{CMC}$ the measurements points of Figure 12 are scattered. This is a consequence of the use of the Du Nouy method for the measurement of $\sigma$ and the difficulty to completely get rid of small bubbles and foaming at the interface when preparing CTAB concentrated SMNs. Figure 12 finally shows that $[\mathrm{CTAB}]_{\mathrm{s}}$ is, as expected, independent of $\left[\mathrm{SiO}_{2}\right]$ and that the saturation molar area is $\Gamma_{\mathrm{s}} \approx 1.510^{-6}$ $\mathrm{mol} / \mathrm{m}^{2}$. This gives an equivalent surface area of $1.1 \mathrm{~nm}^{2}$ consistent with literature ${ }^{37}$ and explains why it is the mixing ratio that gives the correct scaling in Figure 11. 


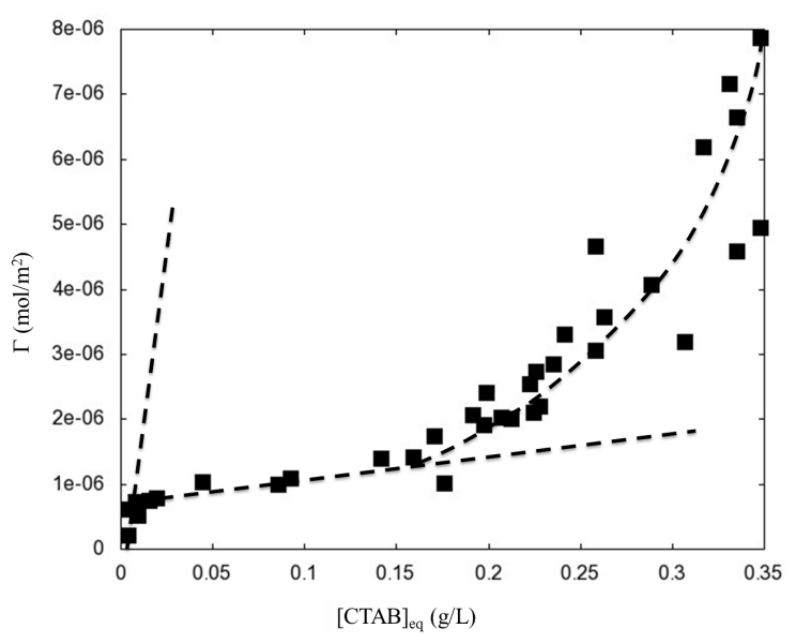

Figure 12: Adsorption isotherm for the measurements points of Figure 11. Dashed lines are plotted to guide eyes.

\subsection{CTAB/SiO $/ 2$ aggregates and morphology of droplets.}

The question is now to justify the use of the above SMN/air results for the description of SMN/PO emulsions. In the concentration domain D2 (Figure 6) SMN/air interfacial tension is close to the one of pure water (Figure 10) and drops when $R=R_{c}$ that is to say when the transition mixing ratio between SDE and PDE is crossed (upper dashed line in Figure 6). One can therefore reasonably assume that emulsification of the SMNs into PO will not strongly modify the $\mathrm{CTAB} / \mathrm{SiO}_{2}$ partition discussed for SMN/air interfaces. Regarding to emulsification of SMNs, air and PO can therefore be considered as having similar properties. This is consistent with the high HLB of CTAB and the low surface activity of $\mathrm{SiO}_{2}$ nanoparticles when surfactant free. The consequence of this is that SMN/PO interfacial properties are mainly driven by SPAN80 as confirmed by the weak dependence of [SPAN80] with the SMN composition when $[\mathrm{CTAB}]<0.2 \mathrm{~g} / \mathrm{L}$ (Figure 9). This last statement explains why the dispersion of the SMNs is improved when increasing [SPAN80] (Figure 7) but actually does not help the understanding of the PDE to SDE structural changes.

To have some insights in this problem, it is necessary to come back one more moment to Figure 6 and to SPAN80 free emulsions. It is clear from this figure that when $[\mathrm{CTAB}]$ is increased, for a given value of $\left[\mathrm{SiO}_{2}\right]$, PDEs become SDEs when the transition line between D2 and D1 is crossed. But this figure also demonstrates that PDEs can already be observed when $[\mathrm{CTAB}]<[\mathrm{CTAB}]_{\mathrm{s}}$ that is to say when $\mathrm{CTAB}$ monomers are still all adsorbed on nanoparticles. The SMN/PO interface remains therefore CTAB free. $\mathrm{SiO}_{2}$ nanoparticles alone are not surface active. The fact that PDEs can be stabilized in such conditions results therefore from the fact that nanoparticles are surface active only when modified by CTAB. In common RamsdenPickering emulsions, particles alone are sufficient to stabilize emulsions but here it is the combined effect of $\mathrm{CTAB}$ and $\mathrm{SiO}_{2}$ nanoparticles that determine emulsion stability. This is consistent with interfacial rheology results 38. As CTAB controls the hydrophobicity of nanoparticles it drives their mutual interaction and their aggregation properties. In principle, $\mathrm{SiO}_{2}$ aggregates result from the balance between van der Waals forces and electrostatic forces. But hydrophobic interactions between CTAB hydrocarbon tails have also to be accounted for since they might overcome Van der Waals forces at short range and make aggregation irreversible ${ }^{32}$. CTAB modified $\mathrm{SiO}_{2}$ nanoparticles give then rise to interfaces where particles behave as a fluid (at low coverage rate) or a solid (when densely packed). At intermediate concentrations, they form interconnected networks with specific visco-elastic properties 31-33. Such interconnected complexes were investigated by Brewster angle microscopy ${ }^{31}$. They appear as thin white skins at SMN/PO interfaces when samples are kept for several hours when SMN and PO are in separated phases.

As CTAB modified $\mathrm{SiO}_{2}$ nanoparticles are surface active, interconnected complexes of $\mathrm{SiO}_{2}$ particles are also surface active. These latter show up as microstructures that have a non-negligible elasticity ${ }^{28}$ and therefore can drastically modify interface curvature as schematically plotted in Figure 13(a). In this figure, stiffness of the microstructures is supposed to be large enough for them not to bend under the effect of capillary forces. It illustrates how the adsorption of $\mathrm{SiO}_{2}$ complexes can generate deformed droplets and therefore how PDEs can be stabilized. In this view only mechanical forces are involved. But chemical processes probably also set in. For example, when CTAB is in large excess $\left([\mathrm{CTAB}]>[\mathrm{CTAB}]_{\mathrm{s}}\right)$ hydrophilicity of the $\mathrm{SiO}_{2}$ microstructures will increase due to the appearance of multilayers. Free monomers and/or aggregates will start to adsorb at the SMN/PO interface. This will promote a reduction of the contact angle of the $\mathrm{SiO}_{2}$ nanoparticles, favor their solubilisation back into the SMN and their replacement by CTAB. Such a situation is illustrated with the aggregate in the left section of the droplet displayed in Figure 13(b). The local curvature of the droplets is then no more constrained by stiff objects and spherical geometries become favorable. Such rearrangements have been experimentally evidenced and shown to involve depletion forces ${ }^{31}$.
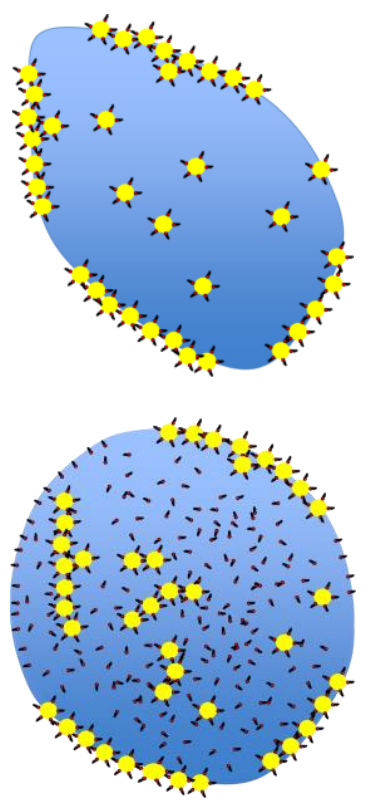

(b)

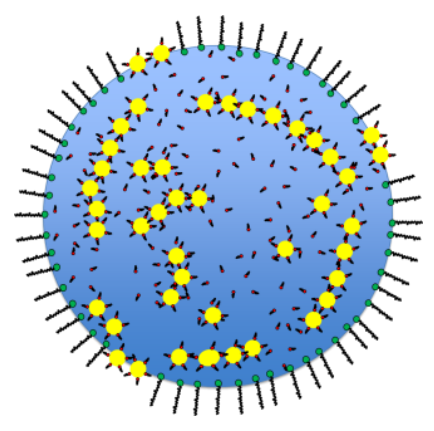


Figure 13: Schematic view of the structure of SNM/PO interfaces for a single droplet. (a) polymorphous droplet with stiff adsorbed $\mathrm{SiO}_{2}$ microstructures and $[\mathrm{SPAN80}]=0 \mathrm{~g} / \mathrm{L}$, (b) almost spherical droplet with flexible microstructures and partial solubilisation (see up left aggregate) still with [SPAN80] $=0 \mathrm{~g} / \mathrm{L}$, (c) spherical droplet with microstructure solubilisation and $\left[\mathrm{SPAN80} \neq 0 \mathrm{~g} / \mathrm{L}\right.$. $\mathrm{SiO}_{2}$ nanoparticles are yellow, CTAB (resp. SPAN80) molecules have red (resp. green) polar head. In (c), SPAN80 molecules solubilized in PO are not plotted.

Now, what about the effect of SPAN80? It was previously shown that the transition concentration $[\mathrm{SPAN} 80]_{\mathrm{c}}$ does not depend on the SMN and that the structure of the emulsions is monitored only by SPAN80 for moderate CTAB concentrations (Figure 9). The above discussion for SPAN80 free emulsions pointed out the possibility to promote changes in the adsorption properties of $\mathrm{SiO}_{2}$ microstructures by desorption phenomena and reduction of visco-elasticity. Similarly to what happens for CTAB, larger values of [SPAN80] will also increase hydrophobic interactions. Due to this, the affinity of $\mathrm{SiO}_{2}$ microstructures with the SMN/PO interfaces can be reduced. In such a scenario, SPAN80 molecules adsorb at the SMN/PO interface, replace adjacent $\mathrm{SiO}_{2}$ microstructures and favour their solubilisation back into the droplets as illustrated in Figure 13(c). SMN/PO interfaces become then microstructure-free and only populated by surfactant molecules. The usual equilibrium properties of surfactant layers are then recovered, droplets get spherical and PDE turn back to usual SDEs.

To our knowledge, the mechanism(s) involved at microscopic scales for the solubilisation of $\mathrm{SiO}_{2}$ microstructures is(are) still to be understood in particular regarding to the precise role of SPAN80 molecules. From this point of view Figure 13(c) is a very naive view and should therefore be considered with care. It is for example not clear whether SPAN80 adsorbs preferentially on the $\mathrm{SMN} / \mathrm{PO}$ interface as displayed in this figure or on $\mathrm{SiO}_{2}$ microstructures or even generate self-emulsification processes or micelles that would solubilize one liquid into the other. In the first case, usual surfactant layers and liquid-

\section{References}

(1) Frelichowska, J.; Bolzinger, M.-A.; Valour, J.-P.; Mouaziz, H.; Pelletier, J.; Chevalier, Y. Pickering W/o Emulsions: Drug Release and Topical Delivery. Int. J. Pharm. 2009, 368 (1-2), 7-15.

(2) Liu, X.; Yi, C.; Zhu, Y.; Yang, Y.; Jiang, J.; Cui, Z.; Jiang, M. Pickering Emulsions Stabilized by Self-Assembled Colloidal Particles of Copolymers of P(St-Alt-MAn)-Co-P(VM-Alt-MAn). J. Colloid Interface Sci. 2010, 351 (2), 315-322.

(3) Li, C.; Liu, Q.; Mei, Z.; Wang, J.; Xu, J.; Sun, D. Pickering Emulsions Stabilized by Paraffin Wax and Laponite Clay Particles. $J$. Colloid Interface Sci. 2009, 336 (1), 314-321.

(4) Yang, Y.; Marshall-Breton, C.; Leser, M. E.; Sher, A. A.; McClements, D. J. Fabrication of Ultrafine Edible Emulsions:

Comparison of High-Energy and Low-Energy Homogenization Methods. Food Hydrocoll. 2012, 29 (2), 398-406.

(5) Dickinson, E. Use of Nanoparticles and Microparticles in the Formation and Stabilization of Food Emulsions. Trends Food Sci.

Technol. 2012, 24 (1), 4-12.

(6) Anton, N.; Vandamme, T. F. Nano-Emulsions and MicroEmulsions: Clarifications of the Critical Differences. Pharm. Res. 2011, 28 (5), 978-985. liquid mechanisms at interfaces prevail while in the second, hydrophobic chain-chain interactions between CTAB and SPAN80 would be the driving mechanism. But several mechanisms most probably contribute simultaneously, promote water solubility and most probably changes in the stiffness of the $\mathrm{SiO}_{2}$ microstructures.

\section{Conclusion}

This work explores the properties of emulsified nanofluids. When emulsifying silica $\left(\mathrm{SiO}_{2}\right)$ aqueous suspensions modified by CTAB in a continuous paraffin oil phase, the morphology of the dispersed phase strongly depends on the $\left[\mathrm{SiO}_{2}\right] /[\mathrm{CTAB}]$ mixing ratio. The droplets can be either spherically shaped as in usual emulsions or consist in polymorphous stiff objects. Similar phenomena are shown to take place for SDS/alumina nanoparticle mixtures. This paper describes the impact of the addition of an oil soluble surfactant (SPAN80) into such emulsions. Their structure is analyzed with optical tomographic microscopy in transmission mode and the flow of images processed for a volume of emulsion of $1 \mathrm{~mm}^{3}$. The specific case of $\mathrm{SiO}_{2} / \mathrm{CTAB}$ mixtures is studied in detail. Interfacial tension measurements are performed to describe the partition of $\mathrm{CTAB}$ for a large range of $\mathrm{SiO}_{2}$ concentrations. They reveal that $\mathrm{CTAB}$ preferentially adsorbs on the nanoparticles in agreement with literature. Important modifications in the structure of the dispersed phase are revealed when [SPAN80] $0.03 \mathrm{~g} / \mathrm{L}$. Below this concentration, droplets are polymorphous while for larger values a usual spherical geometry is recovered. This concentration is shown to depend only weakly of both $\mathrm{CTAB}$ and $\mathrm{SiO}_{2}$ concentrations. A possible mechanism to explain the role of SPAN80 in the structure of the emulsions is finally proposed.

\section{Aknowledgments}

This work was supported by the European Science Foundation COST Actions MP1106 and CM1101, ESA MAP-FASES, CNES and GdR-CNRS MFA.

(7) Schafroth, N.; Arpagaus, C.; Jadhav, U. Y.; Makne, S.; Douroumis, D. Nano and Microparticle Engineering of Water Insoluble Drugs Using a Novel Spray-Drying Process. Colloids Surf. B Biointerfaces 2012, 90, 8-15

(8) Walker, E. M.; Frost, D. S.; Dai, L. L. Particle Self-Assembly in Oil-in-Ionic Liquid Pickering Emulsions. J. Colloid Interface Sci. 2011, 363 (1), 307-313.

(9) Arditty, S.; Schmitt, V.; Giermanska-Kahn, J.; Leal-Calderon, F. Materials Based on Solid-Stabilized Emulsions. J. Colloid Interface Sci. 2004, 275 (2), 659-664.

(10) Köhler, K.; Santana, A. S.; Braisch, B.; Preis, R.; Schuchmann, H. P. High Pressure Emulsification with Nano-Particles as Stabilizing Agents. Chem. Eng. Sci. 2010, 65 (10), 2957-2964.

(11) Sadurní, N.; Solans, C.; Azemar, N.; García-Celma, M. J Studies on the Formation of O/W Nano-Emulsions, by Low-Energy Emulsification Methods, Suitable for Pharmaceutical Applications. Eur. J. Pharm. Sci. 2005, 26 (5), 438-445.

(12) Ramsden, W. Separation of Solids in the Surface-Layers of Solutions and "Suspensions" (Observations on Surface-Membranes, Bubbles, Emulsions, and Mechanical Coagulation). Proc. R. Soc. Lond. 1903, 72 (477-486), 156-164. 
(13) Pickering, S. U. CXCVI.-Emulsions. J. Chem. Soc. Trans. 1907, 91 (0), 2001-2021.

(14) Gautier, F.; Destribats, M.; Perrier-Cornet, R.; Dechezelles, J.F.; Giermanska, J.; Heroguez, V.; Ravaine, S.; Leal-Calderon, F.; Schmitt, V.; Binks, B. P. Pickering Emulsions with Stimulable Particles : From Highly-to Weakly-Covered Interfaces. PCCP 2007, 9 (48), 64556462 .

(15) Binks, B. P.; Clint, J. H.; Whitby, C. P. Rheological Behavior of Water-in-Oil Emulsions Stabilized by Hydrophobic Bentonite Particles (English). Langmuir 2005, 21 (12), 5307-5316.

(16) Melle, S.; Lask, M.; Fuller, G. G. Pickering Emulsions with Controllable Stability. Langmuir 2005, 21 (6), 2158-2162.

(17) Monteux, C.; Jung, E.; Fuller, G. G. Mechanical Properties and Structure of Particle Coated Interfaces: Influence of Particle Size and Bidisperse 2D Suspensions. Langmuir 2007, 23 (7), 3975-3980.

(18) Binks, B. P. Colloidal Particles at Liquid Interfaces. Phys. Chem. Chem. Phys. 2007, 9 (48), 6298-6299.

(19) Arditty, S.; Whitby, C. P.; Binks, B. P. ; Schmitt, V.; LealCalderon, F. Some General Features of Limited Coalescence in SolidStabilized Emulsions. Eur Phys J E Soft Matter Print 2003, 11 (3), 273281.

(20) Eskandar, N. G.; Simovic, S.; Prestidge, C. A. Interactions of Hydrophilic Silica Nanoparticles and Classical Surfactants at Non-Polar Oil-water Interface. J. Colloid Interface Sci. 2011, 358 (1), 217-225.

(21) Binks, B. P.; Rodrigues, J. A.; Frith, W. J. Synergistic Interaction in Emulsions Stabilized by a Mixture of Silica Nanoparticles and Cationic Surfactant. Langmuir 2007, 23 (7), 3626-3636.

(22) Binks, B. P.; Desforges, A.; Duff, D. G. Synergistic Stabilization of Emulsions by a Mixture of Surface-Active Nanoparticles and Surfactant. Langmuir 2007, 23 (3), 1098-1106.

(23) Schmitt-Rozières, M.; Krägel, J.; Grigoriev, D. O.; Liggieri, L.; Miller, R.; Vincent-Bonnieu, S.; Antoni, M. From Spherical to Polymorphous Dispersed Phase Transition in Water/Oil Emulsions. Langmuir 2009, 25 (8), 4266-4270.

(24) Ravera, F.; Ferrari, M.; Liggieri, L.; Loglio, G.; Santini, E.; Zanobini, A. Liquid-liquid Interfacial Properties of Mixed Nanoparticlesurfactant Systems. Colloids Surf. Physicochem. Eng. Asp. 2008, 323 (13), 99-108.

(25) Limage, S.; Schmitt, M.; Vincent-Bonnieu, S.; Dominici, C.; Antoni, M. Characterization of Solid-Stabilized Water/oil Emulsions by Scanning Electron Microscopy. Colloids Surf. Physicochem. Eng. Asp. 2010, 365 (1-3), 154-161.

(26) Lan, Q.; Yang, F.; Zhang, S.; Liu, S.; Xu, J.; Sun, D. Synergistic Effect of Silica Nanoparticle and Cetyltrimethyl Ammonium Bromide on the Stabilization of O/W Emulsions. Colloids Surf. Physicochem. Eng. Asp. 2007, 302 (1-3), 126-135.

(27) Santini, E.; Liggieri, L.; Sacca, L.; Clausse, D.; Ravera, F. Interfacial Rheology of Span 80 Adsorbed Layers at Paraffin Oil-water
Interface and Correlation with the Corresponding Emulsion Properties. Colloids Surf. Physicochem. Eng. Asp. 2007, 309 (1-3), 270-279.

(28) Limage, S.; Krägel, J.; Schmitt, M.; Dominici, C.; Miller, R.; Antoni, M. Rheology and Structure Formation in Diluted Mixed Particle-Surfactant Systems. Langmuir 2010, 26 (22), 16754-16761.

(29) Fouconnier, B.; Román-Guerrero, A.; Vernon-Carter, E. J Effect of [CTAB]-[SiO2] Ratio on the Formation and Stability of Hexadecane/water Emulsions in the Presence of $\mathrm{NaCl}$. Colloids Surf. Physicochem. Eng. Asp. 2012, 400, 10-17.

(30) Binks, B. P.; Whitby, C. P. Nanoparticle Silica-Stabilised Oilin-Water Emulsions: Improving Emulsion Stability. Colloids Surf. Physicochem. Eng. Asp. 2005, 253 (1-3), 105-115.

(31) Maestro, A.; Guzmán, E.; Santini, E.; Ravera, F.; Liggieri, L.; Ortega, F.; Rubio, R. G. Wettability of Silica Nanoparticle-surfactant Nanocomposite Interfacial Layers. Soft Matter 2011, 8 (3), 837-843.

(32) Maestro, A.; Deshmukh, O. S.; Mugele, F.; Langevin, D. Interfacial Assembly of Surfactant-Decorated Nanoparticles: On the Rheological Description of a Colloidal 2D Glass. Langmuir 2015, 31 (23), 6289-6297.

(33) Liggieri, L.; Santini, E.; Guzmán, E.; Maestro, A.; Ravera, F. Wide-Frequency Dilational Rheology Investigation of Mixed Silica nanoparticle-CTAB Interfacial Layers. Soft Matter 2011, 7 (17), 7699.

(34) Schmitt, M.; Limage, S.; Grigoriev, D. O.; Krägel, J.; Dutschk, V.; Vincent-Bonnieu, S.; Miller, R.; Antoni, M. Transition from Spherical to Irregular Dispersed Phase in Water/Oil Emulsions. Langmuir 2014, 30 (16), 4599-4604.

(35) Sasol Germany.

http://www.sasolgermany.de/fileadmin/doc/alumina/DISPERALDISPAL.GB_04.pdf.

(36) Santini, E.; Krägel, J.; Ravera, F.; Liggieri, L.; Miller, R. Study of the Monolayer Structure and Wettability Properties of Silica Nanoparticles and CTAB Using the Langmuir Trough Technique. Colloids Surf. Physicochem. Eng. Asp. 2011, 382 (1-3), 186-191.

(37) Wang, W.; Gu, B.; Liang, L.; Hamilton, W. A. Adsorption and Structural Arrangement of Cetyltrimethylammonium Cations at the Silica Nanoparticle-Water Interface. J. Phys. Chem. B 2004, 108 (45), 1747717483.

(38) Ravera, F.; Santini, E.; Loglio, G.; Ferrari, M.; Liggieri, L. Effect of Nanoparticles on the Interfacial Properties of Liquid/Liquid and Liquid/Air Surface Layers. J. Phys. Chem. B 2006, 110 (39), 1954319551. 